\title{
The Confinement of Electromagnetic Radiation of Nanoemitters in a Multilayered Microsphere with Left-Handed Layers
}

\author{
Gennadiy Burlak, Alfredo Díaz-de-Anda
}

CIICAp, Universidad Autónoma del Estado de Morelos, Cuernavaca, Mexico.

Email: gburlak@uaem.mx

Received October $26^{\text {th }}, 2010$; revised November $23^{\text {rd }}, 2010$; accepted November $29^{\text {th }}, 2010$.

\begin{abstract}
The electromagnetic radiation of nanoemitters placed into a multilayered microsphere with dispersive left-handed $(L H)$ layers included is studied numerically. It is found that in the frequency range where LH layers have a negative refraction index the field frequency spectrum consists of a series of narrow and well separated resonances. In the band of such peaks, the great part of the field energy is located in a LH layer and practically does not leave the microsphere.
\end{abstract}

Keywords: Left-Handed Material, Coated Microsphere, Alternative Multilayered Stack, Numerical Calculations

\section{Introduction}

The recently emerging fields of metamaterials and transformation optics promise a family of exciting applications in nanophotonics with the potential for much faster information processing. The possibility of creating optical negative-index metamaterials (NIM) using nanostructured metal--dielectric composites has triggered intense basic and applied research [1-7]. In very recent experiments [8] it has been demonstrated that the incorporation of gain material in the metamaterial makes it possible to fabricate an extremely low-loss and an active optical NIM that is not limited by the inherent loss in its metal constituent.

Since in such materials the electric field, the magnetic field, and the wave vector of a plane wave form a lefthanded system, they are also called left-handed materials (LHMs). Investigations of the electromagnetic properties of LHMs open various promising directions in the electrodynamics of materials with simultaneously significant electric and magnetic properties, including LHMs.

\section{Multilayered Microspheres}

One of such directions is the use of microcavities and microspheres that provides a new view of various effects and interactions in structured and layered media. Nowadays, the basic regime of the operation of open (uncoated) dielectric microspheres is the whispering gallery mode (WGM) for a microsphere with a radius of the order
$100-1 \mu \mathrm{m}$ or less. The extremely high quality factors ( $Q$-factors) $\sim 10^{8} \div 10^{9}$ have already been realized [9]. But since fabricating the coated dielectric spheres of the submicron sizes, the problem arises of studying the optical oscillations in microspheres beyond the WGM regime for harmonics with small spherical numbers. The peculiarity of such a system (nanoemitter + multilayered microsphere) consists of the following. The ratio of the typical sizes of a nanoemitter $\left(10 \mathrm{~nm}^{9}\right)$ to the typical sizes of a microsphere $(1000 \mathrm{~nm})$ is small $\sim 0.01$. However, the range of the wavelengths of a nanoemitter $\sim 600 \mathrm{~nm}$ is comparable to the width of a layer in the coated microsphere; hence the retardation effects cannot be neglected already.

It is well known that in general, a dielectric sphere has a complex spectrum of the electromagnetic low quality factor eigenoscillations because of the energy leakage into the outer space [10]. The case of the compound structure: the dielectric sphere coated by an alternative stack, is richer. The $Q$-factor of such oscillations strongly depends on the properties of the stack. It has a large value in the frequency regions of high reflectivity, and beyond these regions $Q$ remains small, $[11,12]$. The combination of such factors causes a large variety of optical properties of microspheres with a multilayer stack. In particular, such a system can serve as a spherical symmetric photonic band gap (PGC) structure, which possesses strong selective transmittance properties [13,14], and can arrange the nanometer-sized photon emitters. These possibilities al- 
low us to essentially expand the operational properties of microspheres with the engineering of nanometer-sized photon emitters as attractive artificial light sources for advanced optical technologies. Equally important, this system can provide a compact and simple building block for studying the quantum aspects of light. The attachment of semiconductor nanoclusters onto a spherical microcavity has already allowed the observation of the Rabi splitting [15].

Various properties of the electromagnetic waves in microspheres have been studied both experimentally and theoretically by a number of authors [16-20]. Recently various properties of nanoemitters are discussed (see $[21,22]$ and reference therein).

The incorporation of nanoemitters in the structures with LH materials, e.g. microspheres, can open new possibilities in the electrodynamics of such systems. As far as the author is aware, the radiation of active nanosources, placed in multilayered microspheres with included LH material, has inadequately been studied yet, though it is a logical extension of previous works in the case of bare microspheres. Due to recent synthesization of the microspheres with radial variation in the refractive index [23,24], it is of interest to study new electromagnetic phenomenon when nanosources are incorporated not only onto the surface of a microsphere, but also inside various layers of the spherical stack consisting of the LH and conventional materials.

The analysis of the refractive index properties of optical metamaterials, as a function of real and imaginary parts of dielectric permittivity and magnetic permeability demonstrated a specific interplay between the resonant response of constituents of metamaterials that allows efficient dispersion management. The use of one-dimensional plane structures, including dispersiveless LH layers, allows considerable widening of the band gap of layered structures [25]. Moreover, similarly constructed spherical stack allows extremely narrow frequency resonances in a quasiperiodic structure [26] Nevertheless, up to now, the case of a radiated nanosource placed in a $\mathrm{LH}$ dispersive spherical stack containing both conventional and LH materials has not been studied.

In this paper, we study this situation of a microsphere coated by alternating layers with the dispersive LH layers included. We explored both the frequency and radial dependencies of the field radiation in such a frequency area to answer the question of whether or not the spherical stack can confine the electromagnetic fields and form the new photon states. Our approach is based on the dyadic Green's function (GF) technique that provides an advanced approximation for a multilayered microsphere, in particular in cases where the field is arrested in a LH layer. Such a numerical approach has allowed us to study the multilayered microspheres with any structure of the superficial layers and to evaluate the total contribution of various field states in unified framework.

This paper is organized as follows. In Section 2, our approach and basic equations are formulated for optical fields in a dielectric microsphere coated by a multilayered stack. In Section 3, the properties of permittivity, permeability, and the refractive index of LH layers are discussed. In Section 4, we outline the numerical scheme of applying the GF technique and also discuss our numerical results for the cavity field states radiated by nanoemitters placed into such combined multilayered microsphere. In the last sections, we discuss and summarize our conclusions.

\subsection{Basic Equations the Green Function}

The spatial scale of the nanoemitter objects $(1-100 \mathrm{~nm})$ is at least one order of magnitude smaller than the spatial scale of microspheres $\left(10^{3}-10^{4} \mathrm{~nm}\right)$. Therefore in the coated microsphere (Figure 1), we can represent the nanoemitter structure as a point source placed at $\mathbf{r}^{\prime}$ and having a dipole moment $\mathbf{d}_{0}$. It is well known that the solution of the wave equation for the radiated electromagnetic field $\mathbf{E}$ due to a general source $\mathbf{J}\left(\mathbf{r}^{\prime}\right)$ is [27]

$$
\mathbf{E}(\mathbf{r})=i \omega \mu_{0} \mu \int_{V} d \mathbf{r}^{\prime} \mathbf{G}\left(\mathbf{r}, \mathbf{r}^{\prime}, \omega\right) \cdot \mathbf{J}\left(\mathbf{r}^{\prime}\right),
$$

where $\mathbf{G}\left(\mathbf{r}, \mathbf{r}^{\prime}, \omega\right)$ is the dyadic GF, which depends on the type of boundary conditions imposed on $\mathbf{E}(\mathbf{r})$ and contains all the physical information necessary for describing the multilayered structure (the time dependence is assumed to be $\left.e^{i \omega t}\right)$. Equation (1) is complemented by the standard boundary conditions: limitation of the fields in the center of the microsphere and continuity of the tangential components of the fields at the interfaces of layers. We also use

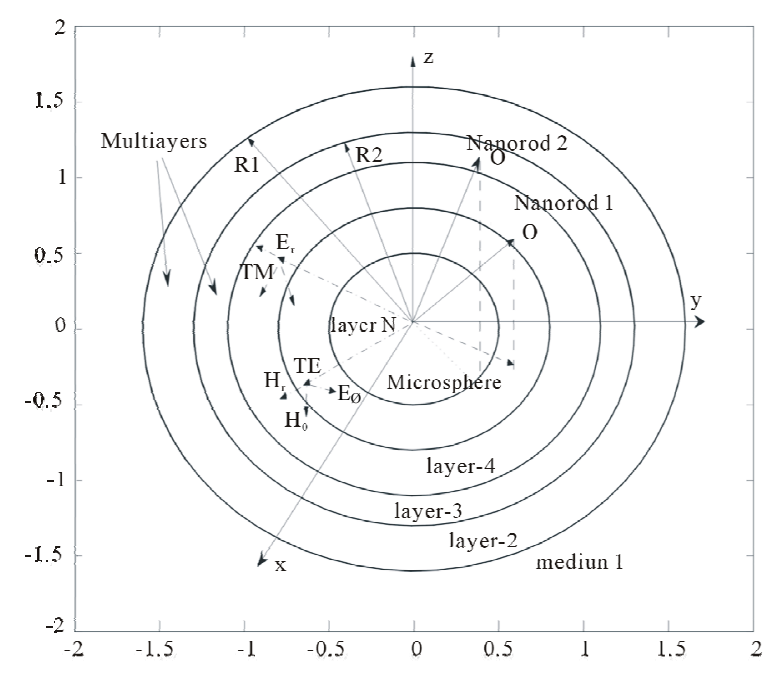

Figure 1. Geometry of a multilayered microsphere. A stack of multilayers with LH layers included is deposited on the surface of the microsphere. 
Sommerfeld's radiation conditions, where there is only an outgoing wave in the external boundary of the microsphere. In this case, the electromagnetic field $\mathbf{E}$ in the coated structure consists of the sum of the waves radiating in the surrounding medium and the multiple wave reflections due to the interfaces between layers. Substituting the nanoemitter source in the form $\mathbf{J}=i \omega \mathbf{d}, \mathbf{d}=\mathbf{d}_{0} \delta\left(\mathbf{r}-\mathbf{r}^{\prime}\right)$ in (1), we obtain

$$
\mathbf{E}\left(\mathbf{r}, \mathbf{r}^{\prime}, \omega\right)=-\mathbf{p}_{0} \mathbf{G}\left(\mathbf{r}, \mathbf{r}^{\prime}, \omega\right)
$$

where $\mathbf{p}_{0}=\left(\mu \mathbf{d}_{0} / \varepsilon_{0}\right)\left(\omega^{2} / c^{2}\right)$ In such a situation, the nanoemitter frequency spectrum is identical to the dyadic GF spectrum. Thus, the equation of the field generated by a nanoemitter assumes the form of the GF $\mathbf{G}\left(\mathbf{r}, \mathbf{r}^{\prime}, \omega\right)$ equation, and is given by [27]

$$
\left[\frac{\omega^{2}}{c^{2}} \varepsilon(\omega) \mu(\omega)-\nabla \times \nabla \times\right] \mathbf{G}\left(\mathbf{r}, \mathbf{r}^{\prime}, \omega\right)=-\delta\left(\mathbf{r}-\mathbf{r}^{\prime}\right),
$$

where $\mathbf{r}$ is the point where the field is observed, while $\mathbf{r}$ is the nanoemitter (point source) location.

Let us consider the multilayered spherical structure: a concentric system of spherical layers contacting with the sphere (concentric stack) deposited onto the surface of the microsphere with nanoemitters placed in such a structure (see Figure 1.). The layers are localized at the distance $R_{k}$ from the center, where $d_{k}=R_{k}-R_{k+1}$ is the width of a $k$-th layer.

\subsection{GF of a Multilayered Microsphere}

Let us first specify some details of the Green's function te- chnique for multilayered microspheres and introduce our notations. Following the approach [28], we write down DGF of such a system as follows:

$$
\mathbf{G}\left(\mathbf{r}, \mathbf{r}^{\prime}, \omega\right)=\mathbf{G}^{V}\left(\mathbf{r}, \mathbf{r}^{\prime}, \omega\right) \delta_{f s}+\mathbf{G}^{(f s)}\left(\mathbf{r}, \mathbf{r}^{\prime}, \omega\right),
$$

where $\mathbf{G}^{V}\left(\mathbf{r}, \mathbf{r}^{\prime}, \omega\right)$ represents the contribution of the direct waves from the radiation sources in the unbounded medium, whereas $\mathbf{G}^{(f s)}\left(\mathbf{r}, \mathbf{r}^{\prime}, \omega\right)$ describes the contribution of the multiple reflection and transmission waves due to the layer interfaces. The dyadic $\mathrm{GF} \mathbf{G}^{V}\left(\mathbf{r}, \mathbf{r}^{\prime}, \omega\right)$ in (4) is given by

$$
\mathbf{G}^{V}\left(\mathbf{r}, \mathbf{r}^{\prime}, \omega\right)=\frac{\hat{\mathbf{r}} \hat{\mathbf{r}}}{k_{3}^{2}} \delta\left(\mathbf{r}-\mathbf{r}^{\prime}\right)+\frac{i k_{3}}{4 \pi} \sum_{q=1,0} \sum_{n=1}^{\infty} \sum_{m=0}^{n} C_{n m} \mathbf{G}_{q, n m}^{V}\left(\mathbf{r}, \mathbf{r}^{\prime}, \omega\right)
$$

with

$$
C_{n m}=\frac{2 n+1}{n(n+1)} \frac{(n-m) !}{(n+m) !}\left(2-\delta_{0 m}\right),
$$

where the prime denotes the nanoemitter coordinates $\left(r^{\prime}, \theta^{\prime}, \varphi^{\prime}\right), n$ and $m$ are spherical and azimuthal quantum numbers, respectively, while $k_{s}$ is the wave number of the medium where the radiated nanoemitters are located. It is worth noting that due to the dyad $\hat{\mathbf{r}} \hat{\mathbf{r}}$, the $\delta$-function in (5) contributes to the radial (longitudinal) part. Due to the equality $\hat{\mathbf{r}} \cdot(\theta \hat{\theta}+\varphi \hat{\varphi})=0$, such a singularity does not contribute to the field (2) for the considered case of a tangential dipole.

The partial dyadic GF $\mathbf{G}_{q, n m}^{V}\left(\mathbf{r}, \mathbf{r}^{\prime}, \omega\right)$ in (5) has a form

$$
\mathbf{G}_{q, n m}^{V}\left(\mathbf{r}, \mathbf{r}^{\prime}, \omega\right)=\left(\begin{array}{c}
\mathbf{M}_{q, n m}^{(1)}\left(\mathbf{r}, k_{s}\right) \mathbf{M}_{q, n m}\left(\mathbf{r}^{\prime}, k_{s}\right)+\mathbf{N}_{q, n m}^{(1)}\left(\mathbf{r}, k_{s}\right) \mathbf{N}_{q, n m}\left(\mathbf{r}^{\prime}, k_{s}\right) \mathbf{r}>\mathbf{r}^{\prime} \\
\left.\mathbf{M}_{q, n m}\left(\mathbf{r}, k_{s}\right) \mathbf{M}_{q, n m}^{(1)}\left(\mathbf{r}^{\prime}, k_{s}\right)+\mathbf{N}_{q, n m}\left(\mathbf{r}, k_{1}\right) \mathbf{N}_{q, n m}^{(1)}\left(\mathbf{r}^{\prime}, k_{s}\right)\right\}, \mathbf{r}<\mathbf{r}^{\prime}
\end{array}\right)
$$

In (7), vectors $\mathbf{M}$ and $\mathbf{N}$ represent $T E$ - and $T M$-waves, respectively, where

$$
\begin{aligned}
\mathbf{M}_{e{ }_{0}}(k)= & \mp \frac{m}{\sin \theta} j_{n}(k r) P_{n}^{m}(\cos \theta)\left(\begin{array}{c}
\sin \\
\cos
\end{array}\right)(m \varphi) \mathbf{e}_{\theta}-j_{n}(k r) \frac{d P_{n}^{m}(\cos \theta)}{d \theta}\left(\begin{array}{c}
\cos \\
\sin
\end{array}\right)(m \varphi) \mathbf{e}_{\varphi}, \\
\mathbf{N}_{e^{\prime}, n m}(k) & =\frac{n(n+1)}{k r} j_{n}(k r) P_{n}^{m}(\cos \theta)\left(\begin{array}{c}
\cos \\
\sin
\end{array}\right)(m \varphi) \mathbf{e}_{r} \\
& +\frac{1}{k r} \frac{d\left[r j_{n}(k r)\right]}{d r}\left[\frac{d P_{n}^{m}(\cos \theta)}{d \theta}\left(\begin{array}{c}
\cos \\
\sin
\end{array}\right)(m \varphi) \mathbf{e}_{\theta} \mp \frac{m}{\sin \theta} P_{n}^{m}(\cos \theta)\left(\begin{array}{c}
\sin \\
\cos
\end{array}\right)(m \varphi) \mathbf{e}_{\varphi}\right],
\end{aligned}
$$

where $j_{n}(x)$ and $h_{n}(x)$ stand for spherical Bessel and Hankel functions respectively, and $P_{n}^{m}(x)$ is the associated Legendre function. For the sake of simplicity, we use in $(8,9)$ and further on, the following short notation

$$
\mathbf{M}_{\substack{e \\ o} m}(k)=\mathbf{M}_{e_{o} n m}(\mathbf{r}, k) \text { and } \mathbf{M}_{e_{o}}^{\prime}(k)=\mathbf{M}_{e_{o} n m}\left(\mathbf{r}^{\prime}, k\right) .
$$

The superscript ${ }^{(1)}$ in (7-15) indicates that in (8) and (9), the spherical Bessel function $j_{n}(x)$ has to be replaced by the first-type spherical Hankel function $h_{n}^{(1)}(x)$.

The scattering GF $\mathbf{G}^{(f s)}\left(\mathbf{r}, \mathbf{r}^{\prime}, \omega\right)$ is written as

$$
\mathbf{G}^{(f s)}\left(\mathbf{r}, \mathbf{r}^{\prime}, \omega\right)=\frac{i k_{s}}{4 \pi} \sum_{q=e, 0} \sum_{n=1}^{\infty} \sum_{m=0}^{n} C_{n m} \mathbf{G}_{q, n m}^{(f, s)}\left(\mathbf{r}, \mathbf{r}^{\prime}, \omega\right)
$$

where $f$ and $s$ denote the layers where the field point and source point are located; $\delta_{f s}$ is the Kronecker symbol and 


$$
\begin{array}{r}
\mathbf{G}_{q, n m}^{(f s)}\left(\mathbf{r}, \mathbf{r}^{\prime}, \omega\right)=\Delta_{n f}\left(\mathrm{M}_{q, n m}^{(1)}\left(k_{f}\right) \mathrm{P}_{M}+\mathrm{N}_{q, n m}^{(1)}\left(k_{f}\right) \mathrm{P}_{N}\right) \\
+\Delta_{1 f}\left(\mathrm{M}_{q, n m}^{(1)}\left(k_{f}\right) \mathrm{Q}_{M}+\mathrm{N}_{q, n m}^{(1)}\left(k_{f}\right) \mathrm{Q}_{N}\right)
\end{array}
$$

with

$$
\begin{aligned}
& \mathrm{P}_{M}=\Delta_{1 s} A_{M}^{f s} \mathrm{M}_{q, n m}^{\prime}\left(k_{s}\right)+\Delta_{N s} B_{M}^{f s} \mathrm{M}_{q, n m}^{\prime}\left(k_{s}\right), \\
& \mathrm{P}_{N}=\Delta_{1 s} A_{N}^{f s} \mathrm{~N}_{q, n m}^{\prime}\left(k_{s}\right)+\Delta_{N s} B_{N}^{f s} \mathrm{~N}_{q, n m}^{(1)}\left(k_{s}\right), \\
& \mathbf{Q}_{M}=\Delta_{1 s} C_{M}^{f s} \mathrm{M}_{q, n m}^{\prime}\left(k_{s}\right)+\Delta_{N s} B_{M}^{f s} \mathrm{M}_{q, n m}^{\prime(1)}\left(k_{s}\right), \\
& \mathbf{Q}_{N}=\Delta_{1 s} C_{M}^{f s} \mathbf{N}_{q, n m}^{\prime}\left(k_{s}\right)+\Delta_{N s} D_{M}^{f s} \mathbf{N}_{q, n m}^{\prime(1)}\left(k_{s}\right),
\end{aligned}
$$

where $\Delta_{f s}=1-\delta_{f s}, \delta_{f s}$ is the Kronecker symbol, $k_{s}=n_{s}(\omega) \omega / c, n(\omega)=\sqrt{\varepsilon_{s}(\omega) \mu_{s}(\omega)}$ is the refraction index of the $s$-layer (see (22) in next Sec.). Frequency dependent coefficients $A_{k}^{f s}(\omega), \quad B_{k}^{f s}(\omega), C_{k}^{f s}(\omega)$ and $D_{k}^{f s}(\omega)$ in (12-15) are defined from the above-mentioned boundary conditions and describe the details of the wave behavior in the interface of the stack layers.

\subsection{The Recursive Calculations of the Scattering Coefficients}

The use of standard boundary conditions yields the relations between $A_{k}^{f s}(\omega), B_{k}^{f_{s}}(\omega), C_{k}^{f_{s}}(\omega)$ and $D_{k}^{f_{s}}(\omega)$ coefficients that are defined by the reflection $R_{j f}^{k}$ and the transmittance $T_{j f}^{k}$ coefficients (in the layer interfaces) and can be written in the following recursive matrix form:

$$
\mathbf{J}_{k}^{f+1, s}-\mathbf{I}_{k}^{f} \cdot \mathbf{J}_{k}^{f, s}+\boldsymbol{\sigma}^{+} \delta_{, f+1, s}-\mathbf{I}_{k}^{f} \cdot \boldsymbol{\sigma}^{-} \delta_{f s}=0,
$$

where $k=M, N, f=1 \ldots N-1$ (in the spherically $\mathrm{N}$ layered medium, see Figure 1.) and

$$
\begin{gathered}
\mathbf{J}_{k}^{f, s}=\left[\begin{array}{ll}
A_{k}^{f, s} & B_{k}^{f, s} \\
C_{k}^{f, s} & D_{k}^{f, s}
\end{array}\right], \mathbf{I}_{k}^{f}=\left[\begin{array}{cc}
1 / T_{F f}^{k} & R_{F f}^{k} / T_{F f}^{k} \\
R_{P f}^{k} / T_{P f}^{k} & 1 / T_{P f}^{k}
\end{array}\right], \\
\boldsymbol{\sigma}^{+}=\left[\begin{array}{ll}
1 & 0 \\
0 & 0
\end{array}\right], \boldsymbol{\sigma}^{-}=\left[\begin{array}{ll}
0 & 0 \\
0 & 1
\end{array}\right] .
\end{gathered}
$$

The explicit expressions for the reflection $R_{j f}^{k}$ and the transmittance $T_{j f}^{k}$ coefficients from (17) are written in the Appendix.

The boundary conditions for the limitation of field in the center of the microsphere and the Sommerfeld's radiation condition at $r \rightarrow \infty$ yield that to (16) one should add the requirement at $f=N, 1$ of the form $A_{k}^{N, s}=B_{k}^{N, s}=0$ and $C_{k}^{1, s}=D_{k}^{1, s}=0$. It is worth noting that due to the spherical symmetry of the system the coefficients $A_{k}^{f, s}, B_{k}^{f, s}, C_{k}^{f, s}$ and $D_{k}^{f, s}$ are functions of $n$ but not of $m$.

\subsection{Permittivity, Permeability, and Refractive Index of LH Layers}

Let us consider a causal linear magnetodielectric medium characterized by a (relative) permittivity $\varepsilon(\mathbf{r}, \omega)$ and a (relative) permeability $\mu(\mathbf{r}, \omega)$, both of which are spatially varying, complex functions of frequency satisfying the relations.

$$
\varepsilon\left(\mathbf{r},-\omega^{*}\right)=\varepsilon^{*}(\mathbf{r}, \omega), \mu\left(\mathbf{r},-\omega^{*}\right)=\mu^{*}(\mathbf{r}, \omega) .
$$

The relation $n^{2}(\mathbf{r}, \omega)=\varepsilon(\mathbf{r}, \omega) \mu(\mathbf{r}, \omega)$ formally offers two possibilities for the (complex) refractive index $n(\mathbf{r}, \omega)$

$$
n(\mathbf{r}, \omega)= \pm \sqrt{|\varepsilon(\mathbf{r}, \omega)(\mathbf{r}, \omega)|} e^{i\left[\varphi_{\varepsilon}(\mathbf{r}, \omega)+\varphi_{\mu}(\mathbf{r}, \omega)\right] / 2},
$$

where

$$
0<\left[\varphi_{\varepsilon}(\mathbf{r}, \omega)+\varphi_{\mu}(\mathbf{r}, \omega)\right] / 2<\pi .
$$

Further, we follow [29] that allow us to rewrite (20) as

$$
n(\mathbf{r}, \omega)=\sqrt{|\varepsilon(\mathbf{r}, \omega) \mu(\mathbf{r}, \omega)|} e^{i\left[\varphi_{\varepsilon}(\mathbf{r}, \omega)+\varphi_{\mu}(\mathbf{r}, \omega)\right] / 2},
$$

In the following, we refer to the material of a layer as being left-handed (or metamaterial) if the real part of its refractive index is negative. In order to allow a dependence on the frequency of the refractive index, let us restrict our attention to a single-resonance permittivity

$$
\varepsilon(\omega)=1+\frac{\omega_{\mathbf{P}_{e}}^{2}}{\omega_{\mathbf{T} e}^{2}-\omega^{2}-i \omega \gamma_{e}},
$$

and a single-resonance permeability

$$
\mu(\omega)=1+\frac{\omega_{\mathbf{P} m}^{2}}{\omega_{\mathbf{T} m}^{2}-\omega^{2}-i \omega \gamma_{m}},
$$

where $\omega_{\mathbf{P} e}, \omega_{\mathbf{P}_{m}}$ are the coupling strengths, $\omega_{\mathbf{T} e}, \omega_{\mathbf{T} m}$ are the transverse resonance frequencies, and $\gamma_{e}, \gamma_{m}$ arethe absorption parameters. Both the permittivity and the permeability satisfy the Kramers-Kronig relations. Figure 2 where $\omega_{\mathbf{P} e}, \omega_{\mathbf{P} m}$ are the coupling strengths, $\omega_{\mathbf{T} e}, \omega_{\mathbf{T} m}$ are the transverse resonance frequencies, and $\gamma_{e}, \gamma_{m}$ are the absorption parameters. Both the permittivity and the permeability satisfy the Kramers-Kronig relations. Figure 2 shows the LH refractive index $n(\mathbf{r}, \omega)=$ $\operatorname{Re} n(\mathbf{r}, \omega)+i \operatorname{Im} n(\mathbf{r}, \omega) \quad(\omega=2 \pi f)$, with the permittivity $\varepsilon(\omega)$ and the permeability $\mu(\omega)$ being respectively given by (23) and (24)) in the frequency interval from $155 \mathrm{THz}$ up to $175 \mathrm{THz}$. In the inset, the details of $n(\mathbf{r}, \omega)$ are shown in the frequency interval from $164 \mathrm{THz}$ up to $175 \mathrm{THz}$ where $\operatorname{Re} n(\mathbf{r}, \omega)<0$. It is worth noting that the negative real part of the refractive index is typically observed together with strong dispersion, so that absorption cannot be disregarded in general. However, in a very recent [8], it was experimentally demonstrated that the incorporation of gain material in a metamaterial makes it possible to fabricate an extremely low-loss and active optical devices. Thus, the original loss-limited negative 


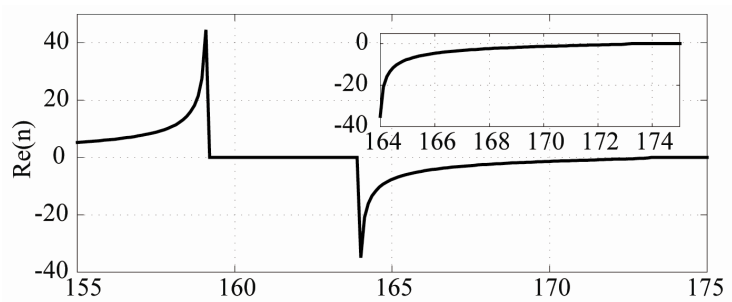

(a)

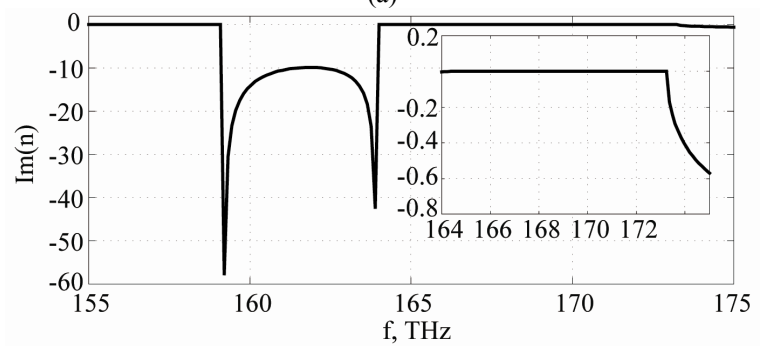

(b)

Figure 2. (Color online.) Real (a) and imaginary (b) parts of the refractive index $n(\omega) \omega=2 \pi f$ of $\mathbf{L H}$ layer in the stack as functions of frequency $f=\omega / 2 \pi$, with the permittivity $\varepsilon(\omega)$ and the permeability $\mu(\omega)$ being respectively given by (23) and (24) $\omega_{T m}=\left(10^{16} / 2 \pi\right) T H z ; \omega_{P m}=0.43 \omega_{T m}$; $\omega_{P e}=0.75 \omega_{T m} ; \gamma_{e}=\gamma_{m}=10^{-7} \omega_{T m}$ (solid lines)]. The values of the parameters have been chosen to be similar to those in [29]. Insets show the details of $\operatorname{Re} n$ and $\operatorname{Im} n$ in the area where $\operatorname{Re} n<0$. See details in text.

refractive index can be drastically improved with loss compensation in the visible wavelength range.

\section{Numerical Results}

Analytical solutions to (16) for scattering coefficients $A_{k}^{f, s}, B_{k}^{f, s}, C_{k}^{f, s}$ and $D_{k}^{f, s}$ for cases of 1 or 2 layers in a spherical stack were derived in [28]. But corresponding equations are rather laborious, and thus are hardly suitable in practice for studying the frequency spectrum for the cases with more than 2 layers in the stack already. However, namely in such a structure, one can expect physically interesting phenomena due to the wave re-reflections in the layers of the stack. Similarly to the plane case, such phenomena are most pronounced when the thicknesses of the alternating spherical layers are approximately equal to $\lambda / 4$ (quarter-wave layers) [11]. In a general case of alternating layers (having small losses), the equality $k_{0}\left|n_{k}\right| d_{k}=\pi / l,\left(d_{k}\right.$ is width, $\left.k_{0}=\omega / c\right)$ is considered, so that $d_{k}=\pi / l k_{0}\left|n_{k}\right|$, where $l$ is integer. In this case, the optical thicknesses of the conventional and LH material layers are the same $d_{1}\left|n_{1}\right|=d_{2}\left|n_{2}\right|$.

\subsection{The Spherical Stack}

We consider a spherical stack with $1 / k_{0}=\Lambda_{0} / 2 \pi$, where $\Lambda_{0}$ is the reference wavelength of the structure. The equality $d_{k}\left|n_{k}\right|=\Lambda_{0} / 4$ corresponds to the quarter-wave case. (Let us remind that the continuity of the fields in the layer interfaces requires the continuity of impedances $Z=(\mu / \varepsilon)^{1 / 2}$ which is positive for both the LH and the conventional layers.) Since the amplitude of a spherical electromagnetic wave depends on the distance to the center of the microsphere, such a $\Lambda_{0} / 4$ approximation is only asymptotically close to the plane wave case. So such a structure can be optimized yet with respect to the local properties of the layers in the stack.

It is worth to identify the nanosource position in a microsphere. If a nanoemitter is placed close to the center of a microsphere, the system is nearly spherically symmetric; therefore the modes with small spherical quantum numbers mainly contribute to the sums in $(5,10)$. This case is close to a rotational invariant geometry where the dipole moment orientation does not need to be specified. Therefore, we draw more attention to a case where the nanoemitter is placed rather far from the center in one of the layers of the spherical stack. In such a system, the preferred direction (center-source) arises, therefore larger numbers of spherical modes contribute to DGF $(5,10)$. As a result the frequency spectrum of DGF becomes richer but more complicated.

\subsection{The Numerical Scheme of Applying the Dyadic Green's Function}

In this section, we numerically explore the details of frequency and radial dependencies of nanoemitter radiation (the dyadic Green's function) for alternating quarter-wave layers deposited on the surface of a microsphere (Figure 1). We use the following steps: 1) we solve (16) for $N$-layered spherical structure; 2) we insert the calculated matrices $A_{k}^{f, s}, B_{k}^{f, s}, C_{k}^{f, s}$ and $D_{k}^{f, s}$ into (12-15), and finally, 3) to obtain the Green's tensor $\mathbf{G}\left(\mathbf{r}, \mathbf{r}^{\prime}, \omega\right)$ (4) we calculate the sums in (5) and (10).

The realization of such a program requires quite intensive computations. Let us outline some details. For a numerical solution, we represent (16) in the form of the matrix equation $F_{i j}(a, b, c, d)=0 \quad(i, j=1,2)$ with respect to the unknown boundary amplitudes $a=A_{k}^{1, s}, \quad b=B_{k}^{1, s}$, $c=C_{k}^{N, s}, \quad d=D_{k}^{N, s}$. Our algorithm has been constructed in such a way, that with the required coefficients $A_{k}^{1, s}, B_{k}^{1, s}, C_{k}^{N, s}, D_{k}^{N, s}$ and the intermediate matrices $\mathbf{J}_{k}^{f, s}$ (which are necessary for computing the fields in the internal layers) have been calculated. Having these matrices calculated, we further make use them in (4-15). Finally, this allows us to calculate the dyadic Green's tensor in any point of the spherical structure and for any number of layers in the stack. Since the complete Green tensor is known, there is, of course, no obstacle to performing the calculations for an arbitrary position of the nanoemitters in a coated microsphere. We note that such a stack in general 
may have an arbitrary or even random structure.

For our numerical approach, the infinite summation in (5) and (10) has to be truncated. The truncation index $n=N_{\max }$ must be chosen, so that a further increase of $n$ does not change the results within a given accuracy. The physical factor, determining the value of spherical numbers $n=N_{\text {max }}$, is the number of the field eigenmodes that contribute to the spectrum of DGF in the frequency range of interest. One can see that the contribution of high-order spherical modes $n$ in (5) and (10) may be weakened due to $C_{n m}$ factor (6). Because of the complexity of the field spatial structure, it is difficult to establish some analytical criterion for $N_{\max }$. In the process of our calculations, we have increased the quantity $N_{\max }$ till the sum in (4) ceases to change essentially. Usually $N_{\max }$ did not exceed $N_{\max } \leq 25-30$. We have found that such an approximation remains working well up to very high spherical numbers (WGM regime). It is worth noting that the continuity of DGF in the layer interfaces is very sensitive to various inaccuracies in calculations and can thus serve as a good criterion for estimating the global accuracy of the results.

Since nanoemitters (e.g. nanorods) are highly polarized objects, we pay more attention to the case where the dipole orientation of the nanoemitter is $\mathrm{d}=d \hat{\varphi}$, so only the tangential components of the Green's tensor $G_{\varphi \varphi}$ contribute.

\subsection{The Parameters of Calculations}

The following parameters have been used in our calculations: the geometry of a system is $A B C B C B C . . B D$, where the letters $A, B, C, D$ indicate the materials in the system, $\Lambda_{0}=1.75 \mu \mathrm{m} \quad K_{0}=2 \pi f_{0} / c, f_{0}=171.5 \mathrm{THz}$. A bottom microsphere has a refraction index $\left(n_{4}=1.5+i 2 \cdot 10^{-4}\right)$ ( $A$, glass, radius $1000 \mathrm{~nm}$ ). The refraction index of the LH layer is given by (22) (see Figure 2 for details) ( $B$, width $437 \mathrm{~nm}), n_{2}=1.46+i 3 \cdot 10^{-3} \quad\left(\mathrm{SiO}_{2}, \mathrm{C}\right.$, width $300 \mathrm{~nm}$ ) and $n_{1}=1$ ( $D$, surrounding space). To consider the realistic layers case we added to each $n_{i}$ a small imaginary part, which corresponds to the material dissipation. We note that even in a material lossless case in such a system (open system), there are losses due to leakage of the field into the surrounding space.

\subsection{The Frequency Spectrum of the GF}

The above-written approach has allowed us to explore both the frequency spectrum and the radial distribution of the Green's function in the multilayered microsphere. The results of our calculations are shown in Figures 3-7

Mainly, we study the case of 7 -layered systems (spherical stack with 7 layers deposited on the surface of the microsphere). In Figure 3(a), we plot the frequency dependence of the imaginary part for the tangential com ponent of GF $G_{\varphi \varphi}\left(r, r^{\prime}, f\right)$ in the source point $\left(r=r^{\prime}\right)$,

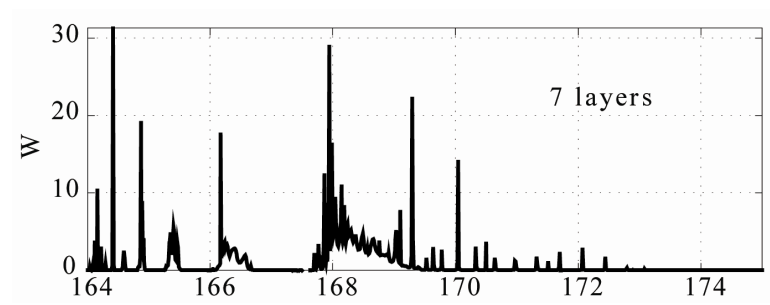

(a)

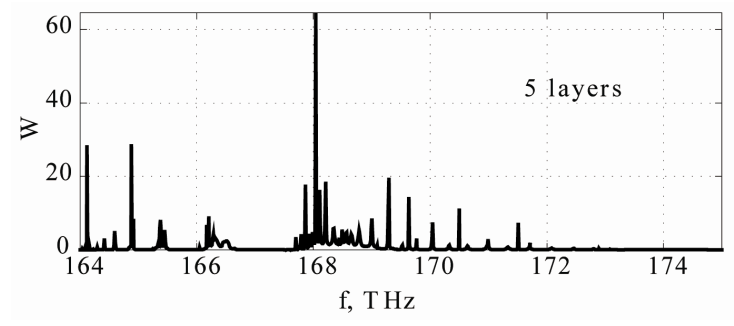

(b)

Figure 3. (Color online.) Frequency spectrum of the imaginary part of the GF $W=\operatorname{Im}\left(G_{\varphi \varphi}(r, r, f)\right)$ (arbitrary units) in the area of $\operatorname{Re}(n<0)$ (see Figure 2(a)), (a) 7 multilayers; (b) 5 multilayers.

where the nanoemitter is located at $r^{\prime}=1480 \mathrm{~nm}$ in $\mathrm{SiO}_{2}$ layer. For comparison, we also present in Figure 3(b) the frequency spectrum of the $\operatorname{Im}\left(G_{\varphi \varphi}\right)$ for a 5 -layer stack. One can see that the structure of the spectrum is similar in both cases.

We observe from Figure 3 (a) that the spectrum of $\operatorname{Im}\left(G_{\varphi \varphi}\right)$ consists of peaks with various amplitudes; the highest peaks are located at $164 \mathrm{THz}$ and $168 \mathrm{THz}$. Theyhave a rather indented form due to the contribution of several close resonances corresponding to various spherical modes. Such peaks have a typical Lorentzian line shape $\operatorname{Im}\left(G_{\varphi \varphi}\right) \sim \gamma /\left(\delta^{2}+\gamma^{2}\right)$, where $\delta$ is a detuning from the resonance and $\gamma$ is the linewidth.

\subsection{The Radial Structure of GF in Spherical Stack}

Some interesting special cases can be considered by analyzing $W(r, f)=\operatorname{Im}\left(G_{\varphi \kappa}\left(r, r^{\prime}=\right.\right.$ const,$\left.\left.f\right)\right)$ as a function of $r$ for a different $f$. In Figure 4, the radial distributions $W(r, f)$ corresponding to the frequency resonances at $164 \mathrm{THz}, 165 \mathrm{THz}$, and $168 \mathrm{THz}$ (solid lines, $r=1480 \mathrm{~nm}$ is the position of a nanoemitter), and the refractive index dependencies of the radial stack (dash lines) are shown. We observe from Figure 4 that the field structure differs considerably for resonances corresponding to the values of the dispersive refractive index of the LH layers $\operatorname{Re} n(f)$. Figure 4(a) shows that for $f=164 \mathrm{THz}$ the field has the form of a resonant oscillation in the LH layer. For $f=168 \mathrm{THz}$ (see Figure 4(c)), the field has shape of a single spatial pulse located in the 
area of the interface of the first $\mathrm{LH}$ layer with a $\mathrm{SiO}_{2}$ layer. The case $f=165 \mathrm{THz}$ Figure $\mathbf{4 ( b ) )}$ is an intermediate case. We observe that in all shown cases, the field is confined by an LH layer and practically does not leave the spherical stack.

It is well known that due to the fluctuation-dissipation theorem the correlation function of the photon states in the absorbing environment for temperature $T$ can be written down with the help of the macroscopic GF as follows [30]

$$
\left\langle\mathbf{E}(r) \mathbf{E}\left(r^{\prime}\right)\right\rangle_{\omega \omega}=\frac{\hbar \omega^{2}}{c^{2}} \operatorname{coth}\left(\frac{\hbar \omega}{2 T}\right) \operatorname{Im}\left(\mathbf{G}\left(r, r^{\prime}, \omega\right)\right) \text {. }
$$

In particular, from (25) one can see that the case $r=r^{\prime}$ yields the inequality $\operatorname{Im}(\mathbf{G}(r, r))>0$ that corresponds to the energy of a fluctuating electromagnetic field $\mathbf{E} \geq(\mathbf{r})$ at small dissipation [30]: $\left\langle\mathbf{E}(\mathbf{r})^{2}\right\rangle \sim \operatorname{Im}(G(\mathbf{r}, \mathbf{r}))$, where

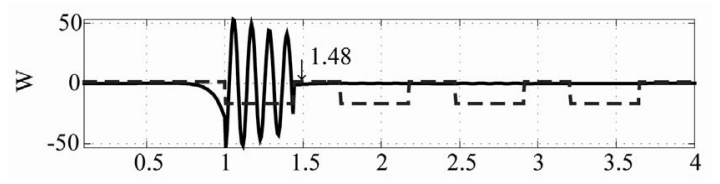

(a)

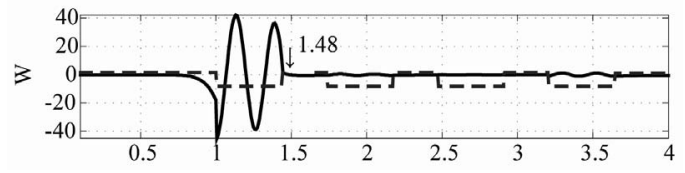

(b)

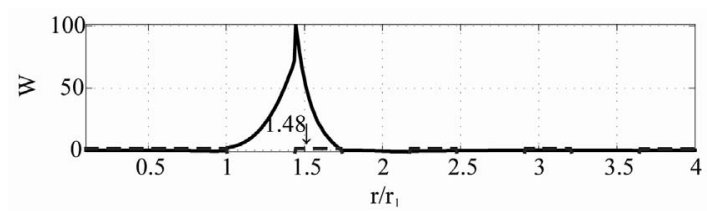

(c)

Figure 4. (Color online.) Details of radial dependence of the imaginary part of the GF $\mathrm{VW}=\operatorname{Im}\left(G_{\varphi \varphi}\left(r, r^{\prime}, f\right)\right)$ (arbitrary units) in the area of $\operatorname{Re}(n)<0$, (see Figure 2 (a)), on frequencies (a) $f=164 \mathrm{THz}, f=165 \mathrm{THz}$, (b), and (c) $f=168 \mathrm{THz}$ (solid line). Radius $r$ is normalized on the radius of the internal microsphere $r_{1}$. The nanosource is placed at $r / r_{1}=1.48$ (small arrow shows location of nanoemitters) in the second layer of the stack. Distribution $\operatorname{Re}(n)$ along the stack is shown by a dashed line; the stack consists of 7 layers. Internal microsphere (glass) occupies the area $r / r_{1}<1$, 1-st layer is LHM with $\operatorname{Re}(n)<0$, then layer $\mathrm{SiO}_{2}$ with $\operatorname{Re}(n)>0$, etc. It is visible that for case (a), there is a confinement of the electromagnetic field already in the first LHM layer. The spatial variation of $W$ becomes less with on increase of the emitter (field) frequency, see case(b). For case (c) only a single field pulse with large amplitude is localized in the first LHM layer. The amplitude of the field decreases appreciably at the increase the radius $r$.
$G(\mathbf{r}, \mathbf{r})$ is the GF, which is $G_{\varphi \varphi}(\mathbf{r}, \mathbf{r})$ in our case. Therefore, the field structure shown in Figure 4(a,b,c) may be treated as a strong correlation of the electromagnetic fields in the vicinity of the LH layer with $\operatorname{Re} n(f)<0$. Figure 5 shows that the field structure (shown in Figure 4 for $r^{\prime}=1480 \mathrm{~nm}$ ) does not change considerably at the shift of the nanoemitter along the layer to $r^{\prime}=1700 \mathrm{~nm}$. The natural question arises, how much can such a stack confine the field energy for a stack with various numbers of layers?

\subsection{GF for Various Numbers of Layers in the Stack}

In Figure 6, the structure $W=\operatorname{Im}\left(G_{\varphi \varphi}\left(r, r^{\prime}, f\right)\right)$ for a system with various layers is shown at frequency $168 \mathrm{THz}$. From Figure 6, we observe that with a change in the number of layers, the spectral peak practically does not variate. This means that for a considered stack (LH layers and conventional layers), the field beyond the first LH layer is so small that the wave boundary re-reflections do not affect the structure of the field in the LH layer. This allows us to conclude that the structure of the spectrum is defined mainly by the intrinsic properties of the spherical stack and weakly depends of the nanoemitter location.

\subsection{The Structure of the GF in a LH Layer}

Furthermore, in some experiments it is important to identify the spatial distribution of the field (for some resonances) radiated by nanosources located in a multilayered microsphere. Therefore, it is of interest to consider the spatial field distribution in a cross-section $(r, \varphi, \theta=$ const $)$

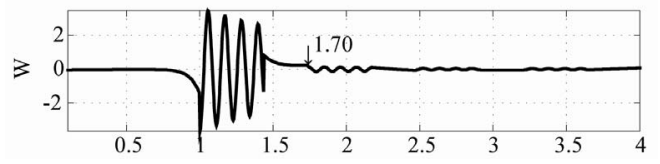

(a)

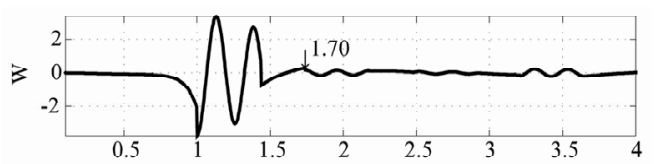

(b)

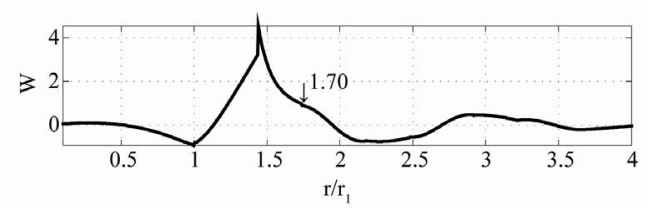

(c)

Figure 5. (Color online.) The same as in Figure 4 but for position of a nanoemitter farther from the center at $r / r_{1}=1.70$. We observe that for such a location of nanosource, the strength $W=\operatorname{Im}\left(G_{\varphi \varphi}\right)$ is less than for case $r / r_{1}=1.48$ shown in Figure 4. 
that contains both the center of the coated microsphere and the nanoemitter. Taking into account the physical meaning of (25), it is of interest to calculate the DGF spectrum fortwo nanoemitters placed at points $r$ and $r^{\prime}$ in a multicoated microsphere for a resonant frequency. In Figure 7, such a distribution is shown for $f=168 \mathrm{THz}$ (see Figure 4(c)).

From (25), one can see that $\operatorname{Im}\left(G_{\varphi \varphi}(r, r, f)\right)$ is propor-

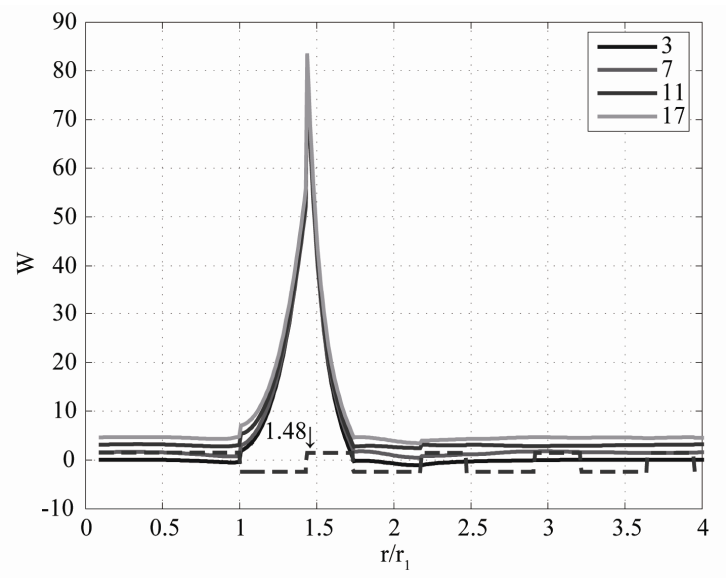

Figure 6. (Color online.) Radial dependencies of imaginary part of the GF $W=\operatorname{Im}\left(G_{\varphi \varphi}\left(r, r^{\prime}, f\right)\right)$ (arbitrary units), in area of $\operatorname{Re}(n)<0$ (see Figure 2(a)), at frequency $f=168 \mathrm{THz}$ for different numbers of layers in the spherical stack. A small shift was added to every line to separate the corresponding close curves. The dashed line shows the radial dependencies of $n$ in the stack.

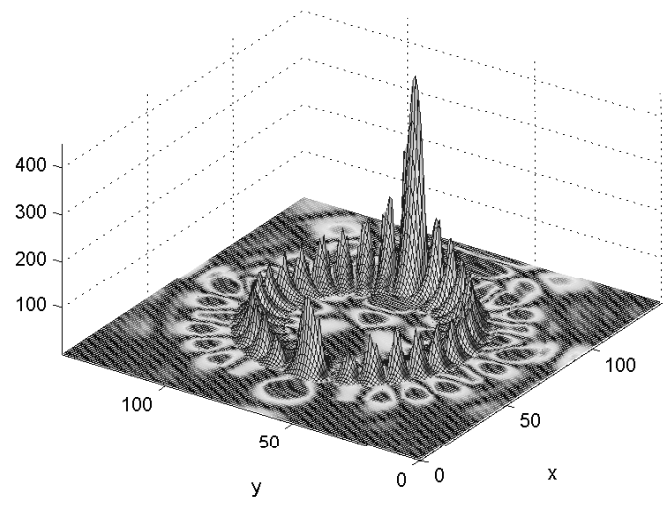

Figure 7. (Color online.) Spatial structure $|W|$ (arbitrary units), $W(r, \varphi)=\operatorname{Im}\left(G_{\varphi \varphi}\left(r, r^{\prime}, \varphi\right)\right)$ in a cross-section $0<r<4000 \mathrm{~nm}$ and $0<\varphi<2 \pi$ of a coated microsphere with $\lambda / 4$ stack for eigenfrequency $f=168 \mathrm{THz}$. A nanoemitter is placed at point $r=1480 \mathrm{~nm}$. The other parameters are the same as in Figure 4. One can observe the confinement of the field in the area of the LH layer of the stack. See details in text. tional to the energy of the fluctuating electromagnetic field in the position of a nanoemitter, while $\operatorname{Im}\left(G_{\varphi \varphi}\left(r, r^{\prime}, f\right)\right)$ corresponds to the correlations of the photonic states at $r$ and $r^{\prime}$. We observe from Figure 7 that the spatial distribution $\operatorname{Im}\left(G_{\varphi \varphi}\left(r, r^{\prime}, f\right)\right)$ has well-defined peaks not only at $r=r^{\prime}$, but also at angles $\varphi_{i}$ along the circle where $r=r^{\prime}$. This means that a strong correlation of field states produced by two separated nanoemitters can be arisen at such a resonance. It is worth to note that such a field state is not a photonic state in general but a state of the macroscopic medium, dressed by the electromagnetic field [30]. We also observe from Figure 7 that the field structure inside of a alternating stack is anisotropic and quite intricate, but the field amplitudes beyond the coated microsphere are small. Nevertheless, a spectral detector placed outside the microsphere still enables a noncontact monitoring of the properties of a nanoemitter located inside a multicoated microsphere.

\subsection{Discussion}

We observe that the use of the of GF technique allows a clear description and a self-consistent understanding of the physics of the electromagnetic phenomena in the spherical stack with LH layers included with respect of the standard decompositions on the spherical modes of the system. We have shown that the difference in the location of a nanosource may lead to the essentially distinct wave pictures. As a result the frequency spectrum of radiations becomes rather indented as it is defined by the sum of the various spherical harmonics with the frequency depending field amplitudes. Such a spectrum differs sufficiently from the case of a spherical stack with conventional materials. Therefore the behavior of the considered compound system is instructively to compare with a limit infinite plane alternating case that has an analytical solution.

The latter stack consists of two different materials with widths $a_{i}$ and impedances $\eta_{i}=\left(\mu_{i} / \varepsilon\right)^{1 / 2}, i=1,2$, where both $\mu_{i}$ and $\varepsilon_{i}$ are dispersiveless quantities. The first layer is a conventional dielectric, while the second layer may be LH material with $\varepsilon<0$ and $\mu<0$. The dispersion relation with $\omega=2 \pi f$ versus the Bloch parameter $\delta(\omega)$ for a $1 D$ case can be written as (see e.g. [25])

$$
\cos (\delta)=\cos (p) \cos (\alpha p)-q \sin (p) \sin (\sigma \alpha p),
$$

with $q=(1 / 2)\left(\eta_{1} / \eta_{, 2}+\eta_{2} / \eta_{1}\right) \geq 1, \quad p=\omega n_{1} a_{1} / c, \quad \alpha=$ $n_{2} a_{2} / n_{1} a_{1}, \sigma= \pm 1$, where $\sigma=-1$ for the LH material, and $n_{i}$ are the refractive indices. The equation (26) is solved for $\delta$ to determine the forbidden bands. Here the negative sign of the refractive index is shown explicitly (by means of parameter $\sigma$ ), so in (26) both refractive indices $n_{1,2}$ are all positive. For quarter-wave stack $\alpha=1$ and $\sigma=1$ (conventional layer) (26) is reduced to the following 


$$
\cos (\delta)=\cos ^{2}(p)-q \sin ^{2}(p),
$$

where $p=\pi f / 2 f_{0}, f_{0}=1 / \Lambda_{0}$ is the reference frequency of the alternating stack) that has well-known solution to define the properties of the band gaps for a conventional $\lambda / 4$ dielectric plane stack, see e.g. Chap. 6 in [31]. However, for $\sigma=-1$ (LH layer) in contrast to $\sigma=1$ case, the equation

$$
\cos (\delta)=\cos ^{2}(p)+q \sin ^{2}(p),
$$

has a real solution for $\delta$ only for $p=l \pi$ or $f=f_{l}=2 l f_{0}$ $(l=1,2, \ldots)$ independently of material impedances (parameter $q$ ). This means that in (27) the transmittance bands shrink to zero [25]. This conclusion is changed for a quasiperiodic spherical stack, where the extremely narrow transmittance peaks are generated [26]. However, for considered here dispersive (with losses) LH case the reference stack frequency $f_{0}=171.5 \mathrm{THz}$ such an area $f_{l}$ with high transmittance is far from the working frequency range of $f<175 \mathrm{TGz}$, where $\operatorname{Re} n$ is negative, see Figure 2. Thus, considered here the frequency range corresponds to a forbidden gap of the infinite plane stack with LH layers included, so the confinement of the electromagnetic field by the LH layer can be expected. Furthermore, the GF technique used here allows not only to identify the layers in which such a confinement occurs, but yet to explore the spatial structure of the field in such an area, see Figure 4 and Figure 7.

\section{Conclusions}

We have studied the frequency spectrum and spatial dependences of the electromagnetic field (the dyadic Green's function), radiated by a nanoemitters (considered in a dipole model) placed into the multilayered microsphere coated by the alternative quarter-wave spherical stack with LH layers included. We found that in the frequency range where LH layers have negative refraction index the frequency spectrum of the field consists of the series of narrow and well separated peaks. In the area of such resonances the LH layers can confine the field of nanoemitters; as a result, the photon states become located in the coated microsphere and practically do not leak into a surrounding space. As the width of resonant peaks is rather small, it allows creating highly polarized nanoemitters oscillating with high $Q$ factor to actively control the polarization state of microcavity photons. Such states can be useful not only for the operational purposes, but also for quantum information technologies.

\section{Acknowledgements}

The work of G.B. is partially supported by CONACYT grant No. 25564.

\section{REFERENCES}

[1] V. G. Veselago, "The Electrodynamics of Substances with Simultaneously Negative Values of $\varepsilon$ and $\mu$," Soviet Physics Uspekhi, Vol. 10, No. 509, 1968, pp. 509-514.

[2] J. B. Pendry, D. Schurig and D. R. Smith, "Controllin Elctromagnetic Fields," Science, Vol. 312, No. 5781, 2006 , pp. 1780-1782.

[3] C. M. Soukoulis, S. Linden and M. Wegener, "Negative Refractive Index at Optical Wavelengths," Science, Vol. 315, No. 5808, 2007, pp. 47-49.

[4] J. Valentine, et al., "Three-Dimensional Optical Metamaterial with a Negative Refractive Index," Nature, Vol. 455, No. 7211, 2008, pp. 376-379.

[5] S. Zhang, et al., "Experimental Demostration of NearInfrared Negative-Index Metamaterial," Physical Review Letters, Vol. 95, No. 13, 2005, pp. 137404-137408.

[6] V. M. Shalaev, "Optical Negative-Index Material," Nature Photonics, Vol. 1, 2006, pp. 41-48.

[7] K. L. Tsakmakidis, A. D. Boardman and O. Hess, "Trapped Rainbow Storage of Light in Metamaterials," Nature, Vol. 450, No. 7168, 2007, pp. 397-401.

[8] S. Xiao, V. P. Drachev, A. V. Kildishev, et.al., "Loss-Free and Active Optical Negative-Index Metamaterial," Nature, Vol. 466, No. 7307, 2010, pp. 735-738.

[9] V. B. Braginsky, M. L. Gorodetsky and V. S. Ilchenko, "Quality-Factor and Nonlinear Properties of Optical Whispering-Gallery Modes," Physics Letters A, Vol. 137, 1989, No. 7-8, pp. 393-397.

[10] J. A. Stratton, "Electromagnetic Theory," McGraw-Hill, New York, 1941.

[11] K. G. Sullivan and D. G. Hall, "Radiation in Spherically Symmetric Structures. I. The Couple-Amplitude Equations for Vevtor Spherical Waves," Physics Review A, Vol. 50, 1994, p. 2701

[12] G. Burlak. "The Classical and Quantum Dynamics of the Multispherical Nanostructures," Imperial College Press, London, 2004.

[13] C. T. Chan, W. Y. Zhang, Z. L. Wang et.al. "Photonic Band Gaps from Metallo-Dielectric Spheres," Physica B., Vol. 279, No. 1-3, 2000, pp. 150-154.

[14] H. Miyazaki, H. Miyazaki, K. Ohtaka, et.al., "Photonic Band in Two-Dimensional Lattices of Micrometer-Sized Spheres Mechanically Arranged under a Scanning Electron Microscope," Journal of Applied Physics, Vol. 87, No. 10, 2000, pp. 7152-7158.

[15] N. Le Thomas, U. Woggon, O. Schops, M. V. Artemyev, M. Kazes and U. Banin, "Cavity QED with Semiconductor Nanocrystal," Nano Letters, Vol. 6, No. 3, 2006, pp. 557561.

[16] A. Moroz, "A Recursive Transfer-Matrix Solution for Adipole Radiating Inside and Outside a Stratified Sphere," Annals of Physics, Vol. 315, No. 2, 2005, pp. 352-418.

[17] P. Voarino, C. Deumie and C. Amra, "Optical Properties Calculated for Multidielectric Quarter-Wave Coatings on Microspheres," Optics Express, Vol. 12, No. 19, 2004, p. 
4476.

[18] C.-C. Chen, Y.-L. Tsai, C.-L. Hsu, et.al., "Propagation Loss Reduction of Photonic Crystal Slab Waveguides by Microspheres," Optics Express, Vol. 12, No. 17, 2004, p. 3934.

[19] H. T. Miyazaki, H. Miyazaki, Y. Jimba, et.al., "Light Diffraction from a Bilayer Lattice of Mocrospheres Enhaced by Specular Resonance," Journal of Applied Physics, Vol. 95, No. 3, 2004, pp. 793-805.

[20] Y. Z. Long, Z. J. Chen, Y. J. Ma et.al., "Electrical Conductivity of Hollow Polyaniline Microspheres Synthesized by a Sel-Assembly Method," Applied Physics Letters, Vol. 84, No. 12, 2004, p. 2205.

[21] N. Le Thomas, E. Herz, O. Schops, U. Woggon and M. V. Artemyev, "Exciton Fine Structure in Single CdSe Nanorods," Physical Review Letters, Vol. 94, No. 1, 2005, pp. 016803-016807.

[22] M. Manghi , X. Schlagberger and R. R. Netz, "Propulsion with a Rotating Elastic Nanorod," Physical Review Letters, Vol. 96, No. 6, 2006, pp. 068101-068105.

[23] A. Petukhova, A. S. Paton, Z. Wei, et.al., "Polymer Multilayer Microspheres Loaded with Semiconductor Quantum Dots," Advanced Functional Materies, Vol. 18, No. 13, 2008, pp. 1961-1968.

[24] A. Petukhova, A. S. Paton, I. Gourevich, et.al., "Hybrid Microspheres with Alternating Layers of Apolimer and

\section{Appendix}

In the above was used the coefficients reflection and transmittance in the spherical layer interfaces without detailed representation. In this Appendix such a representation for coefficients reflection $R_{i f}^{k}$ and transmittance $T_{i f}^{k}$ ( $k=M, N, i=P, F$ ) throw the spherical stack is written as [28],

$$
\begin{aligned}
R_{P f}^{M} & =\frac{k_{f+1} H_{(f+1) f}^{\prime} H_{f f}-k_{f} H_{f f}^{\prime} H_{(f+1) f}}{k_{f+1} J_{f f} H_{(f+1) f}^{\prime}-k_{f} J_{f f}^{\prime} H_{(f+1) f}}, \\
R_{F f}^{M} & =\frac{k_{f+1} J_{(f+1) f}^{\prime} J_{f f}-k_{f} J_{f f}^{\prime} J_{(f+1) f}}{k_{f+1} J_{(f+1) f}^{\prime} H_{f f}-k_{f} J_{(f+1) f} H_{f f}^{\prime}}, \\
R_{P f}^{M} & =\frac{k_{f+1} H_{(f+1) f}^{\prime} H_{f f}-k_{f} H_{f f}^{\prime} H_{(f+1) f}}{k_{f+1} J_{f f} H_{(f+1) f}^{\prime}-k_{f} J_{f f}^{\prime} H_{(f+1) f}}, \\
R_{F f}^{N}= & \frac{k_{f+1} J_{(f+1) f} J_{f f}^{\prime}-k_{f} J_{f f} J_{(f+1) f}^{\prime}}{k_{f+1} J_{(f+1) f} H_{f f}^{\prime \prime}-k_{f} J_{(f+1) f}^{\prime} H_{f f}}, \\
T_{P f}^{M}= & \frac{k_{f+1}\left(J_{(f+1) f} H_{(f+1) f}^{\prime}-J_{(f+1) f}^{\prime} H_{(f+1) f}\right)}{k_{f+1} J_{f f} H_{(f+1) f}^{\prime}-k_{f} J_{f f}^{\prime} H_{(f+1) f}}, \\
T_{F f}^{M}= & \frac{k_{f+1}\left(J_{(f+1) f}^{\prime} H_{(f+1) f}-J_{(f+1) f} H_{(f+1) f}^{\prime}\right)}{k_{f+1} J_{(f+1) f}^{\prime} H_{f f}-k_{f} J_{(f+1) f} H_{f f}^{\prime}},
\end{aligned}
$$

Metal Nanoparticles," Canadian Journal of Chemistry, Vol. 88, No. 3, 2010, pp. 298-304.

[25] I. S. Nefedov and S. A. Tretyakov, "Photonic Band Gap Struture Containg Metamaterial with Negative Permittivity and Permeability," Physical Review E, Vol. 66, No. 3, 2002, pp. 036611-036616.

[26] G. Burlak, A. Diaz-de-Anda, R. S. Salgado and J. P. Ortega, "Narrow Transmittance Peaks in a Multilayered Microsphere with a Quasiperiodic Left-Handed Stack," Optics Communications, Vol. 283, No. 19, 2010, pp. 3569-3577.

[27] J. D. Jackson. "Classical Electrodynamics," John Willey and Sons, New York, 1975.

[28] L.-W. Li, P.-S. Kooi, M.-S. Leong and T.-S. Yeo, "Electromagnetig Dyadic Green's Function in Spherically Multilayered Media," IEEE Transactions on Microwave Theory and Techniques, Vol. 42, No. 12, 1994, pp. 2302-2310.

[29] H. T. Dung, S. Y. Buhmann, L. Knoll, et. al., "Electromagnetic-Field Quantization and Spontaneous Decay in Left-Handed Media," Physical Review A, Vol. 68, No. 4, 2003, pp. 043816-043831.

[30] L. D. Landau and E. M. Lifschitz. "Statistical Physics, Part 2," Pergamon Press, Oxford, England, 1981.

[31] A. Yariv and R. Yeh, "Optical Waves in Crystalls," WilleyInterscience, New York, 2002.

$$
\begin{gathered}
T_{P f}^{N}=\frac{k_{f+1}\left(J_{(f+1) f}^{\prime} H_{(f+1) f}-J_{(f+1) f} H_{(f+1) f}^{\prime}\right)}{k_{f+1} J_{f f}^{\prime} H_{(f+1) f}-k_{f} J_{f f} H_{(f+1) f}^{\prime}}, \\
T_{F f}^{N}=\frac{k_{f+1}\left(J_{(f+1) f} H_{(f+1) f}^{\prime}-J_{(f+1) f}^{\prime} H_{(f+1) f}\right)}{k_{f+1} J_{(f+1) f} H_{f f}^{\prime}-k_{f} J_{(f+1) f}^{\prime} H_{f f}},
\end{gathered}
$$

with

$$
\begin{aligned}
& J_{i l}=j_{n}\left(k_{i} R_{l}\right), \\
& H_{i l}=h_{n}^{(1)}\left(k_{i} R_{l}\right), \\
& J_{i l}^{\prime}=\left.\frac{1}{\rho} \frac{d\left[\rho j_{n}(\rho)\right]}{d \rho}\right|_{\rho=k_{i} R_{l}}, \\
& H_{i l}^{\prime}=\left.\frac{1}{\rho} \frac{d\left[\rho h_{n}^{(1)}(\rho)\right]}{d \rho}\right|_{\rho=k_{i} R_{l}},
\end{aligned}
$$

Here $j_{n}(x)$ and $h_{n}(x)$ stand for spherical Bessel and Hankel functions respectively, $k_{i}$ is the wave vector in the $i$-th material, while $R_{l}$ is the radius of the $i$-th layer. From (28-35) we observe that if the parameters of neighbors $f$ and $(f+1)$ layers are close then the reflection of such $f,(f+1)$ interface is small $R_{\text {if }}^{k} \approx 0$ 